\title{
A SYMMETRICAL CONDITION FOR CO-APOLAR TRIADS ON A CUBIC CURVE
}

\author{
By Williami P. Milne, M.A., D.Sc.
}

[Received March 23rd, 1917.-Read April 19th, 1917.]

\section{Introduction.}

Two triads of points $A B C, D E F$ situated on a cubic curve are said to be "co-apolar" if any point $P$ on the curve possesses the property that the pencils $P[A B C]$ and $P[D E F]$ are apolar. I have shewn in the Proc. London Math. Soc., Ser. 2, Vol. 9, pp. 235-243, that the triads $A B C, D E F$ cannot be arbitrarily chosen on the cubic, but must each be of a special type to which the name "apolar triad" was given. An apolar triad is such that the bi-polar line of any two of its constituent points passes through its third point. In the foregoing paper, I found the necessary and sufficient condition that two apolar triads should be co-apolar, but the form of the condition was unsymmetrical with respect to the two given triads. Inasmuch as the problem under investigation is essentially one of symmetry, it is desirable to obtain a solution in which the given triads are similarly involved. Such is the purpose of the present communication.

The essence of the method lies in projecting the two given triads through a suitably chosen point of the curve on to the curve again so as to obtain two triads lying on the same conic, and thereafter to express the required condition in terms of the covariant geometry of two. triads of points on a conic.

\section{A Pencil of Cubic Curves.}

Let $A B C$ and $D E F$ be two triads on a cubic curve, and let them be projected through a point $T$ of the curve on to the curve again, thus giving the triads $A^{\prime} B^{\prime} C^{\prime}$ and $D^{\prime} E^{\prime} F^{\prime}$. It was proved in the abovementioned paper that, if $A B C$ and $D E F$ be two apolar triads which stand in the co-apolar relation, then $A^{\prime} B^{\prime} C^{\prime}$ and $D^{\prime} E^{\prime} F^{\prime \prime}$ will also be two apolar- 
triads co-apolarly related. It will be convenient if we choose $T$ so that $A^{\prime} B^{\prime} C^{\prime}, D^{\prime} E^{\prime} F^{\prime}$ lie on the same conic $\Gamma$. The positions of $T$ are easily found in terms of elliptic parameters: for, if $A, B$, \&c., represent the elliptic parameters of the points $A, B$, \&c., we have $A+T+A^{\prime} \equiv 0$, \&c., and $\Sigma A^{\prime} \equiv 0$, whence $\Sigma A+6 T=0$, giving thirty-six positions of $T$. Let us consider next the pencil $\Psi$ of cubics circumscribing the triangles $A^{\prime} B^{\prime} C^{\prime}, D^{\prime} E^{\prime} F^{\prime \prime}$ and apolar to them. One cubic of the system is the degenerate cubic consisting of $\Gamma$ and $L$, where $L$ is the line joining the poles of $\Gamma$ with respect to the triangles $A^{\prime} B^{\prime} C^{\prime}$ and $D^{\prime} E^{\prime} F^{\prime \prime}$. It is therefore evident that the nine "associated" points, common to all the members of the pencil $\Psi$, consist of the triads $A^{\prime} B^{\prime} C^{\prime}, D^{\prime} E^{\prime} F^{\prime}$ and three other points $U^{\prime} V^{\prime} W^{\prime}$, which lie on $L$, since $A^{\prime} B^{\prime} C^{\prime}, D^{\prime} E^{\prime} F^{\prime}$ lie on the conic $\Gamma$. Furthermore, $I$ have proved in the Proceedings of the Edin. burgh Mathematical Society, Vol. 30, Session 1911-12, that if a pencil of cubics circumscribe two triangles and be apolar to them, the remaining three points common to all the curves of the system form also a triangle apolar to every member of the system. In the above case, therefore, since $U^{\prime}, V^{\prime}, W^{\prime}$ form a linear triad apolar to every member of the $\Psi$. pencil, we find that the line $L$ joining $U^{\prime}, V^{\prime}, W^{\prime}$ must touch the Cayleyan of every member of the $\Psi$-pencil, as was proved in the aforementioned communication to the London Mathematical Society, for the case of linear apolar triads. The tangents at $U^{\prime}, V^{\prime}, W^{\prime}$ to any member of the $\Psi$-pencil are therefore concurrent; and the locus of the point of concurrency is a straight line $M$, since a $(1,1)$ correspondence exists between the tangents at $U^{\prime}, V^{\prime \prime}$ (or $W^{\prime}$ ), and $U^{\prime} V^{\prime}$ (regarded as a ray from $U^{\prime}$ ) corresponds to $V^{\prime} U^{\prime}$ (regarded as a ray from $V^{\prime}$ ) for the case of the cubic $\Gamma L$. It is easy to identify the line $M$ by considering two particular members of the $\Psi$-systen, viz. the apolar loci of $\left[A^{\prime} B^{\prime} C^{\prime}\right.$, $\left.U^{\prime} V^{\prime} W^{\prime}\right]$ and $\left[D^{\prime} E^{\prime} F^{\prime}, U^{\prime} V^{\prime} W^{\prime}\right]$. I proved (Proc. London Math. Soc., loc. cit.) that the three tangents at $U^{\prime}, V^{\prime}, W^{\prime}$ to the above apolar loci meet at the poles of the line $L$ with respect to the triangles $A^{\prime} B^{\prime} C^{\prime}$, $D^{\prime} E^{\prime} F^{\prime}$ respectively. Hence $M$ is the line joining the poles of $L$ with re. spect to the triangles $A^{\prime} B^{\prime} C^{\prime}, D^{\prime} E^{\prime} F^{\prime}$. It is noteworthy that the poles of $L$ with respect to these two triangles lie on the conic $\Gamma$, since the poles of $\Gamma$ with respect to the triangles lie on $L$.

\section{The Required Condition.}

We thus see that, in virtue of the fact that the tangents at $U^{\prime} V^{\prime} W^{\prime}$ are concurrent on $M$ for every member of the $\Psi$-pencil of cubics, we have established a $(1,1)$ correspondence between the members of the $\Psi$-system 
and the points on the line $M$. We wish to obtain now the point $Q$ on $M$ that corresponds to the apolar locus of $\left[A^{\prime} B^{\prime} C^{\prime}, D^{\prime} E^{\prime} F^{\prime}\right]$, i.e. the point on the line $M$ at which meet the tangents at $U^{\prime}, V^{\prime}, W^{\prime}$ to the above apolar locus.

Take the conic $\mathrm{I}^{\prime}$ in the form $x=t^{2}, y=1, z=2 t$, where the tangents at the Hessian points of $A^{\prime} B^{\prime} C^{\prime}$ and the line joining these points form the triangle of reference. Also let us take $D^{\prime} E^{\prime} F^{\prime \prime}$ in the form

$$
a_{0} t^{3}+3 a_{1} t^{2}+3 a_{2} t+a_{3}=0 .
$$

Let $H$ and $K$ be the poles of the conic $\Gamma$ with respect to the triangles $A^{\prime} B^{\prime} C^{\prime}$ and $D^{\prime} E^{\prime} F^{\prime}$ respectively.

A cubic of the $\Psi$-pencil other than $\Gamma L$ is

$$
\Delta \equiv 8 a_{0} x^{3}+8 a_{3} y^{3}+\left(a_{0}+a_{3}\right) z^{3}+12 a_{1} x^{2} z+12 a_{2} y^{2} z+6 a_{2} z^{2} x+6 a_{1} z^{2} y=0 \text {. }
$$

The apolar locus of $A^{\prime} B^{\prime} C^{\prime}$ and $D^{\prime} E^{\prime} F^{\prime}$ is easily found to be

$$
\left(a_{0}-a_{3}\right) \Delta=18 \mathrm{\Gamma} L,
$$

where

$$
\Gamma \equiv z^{2}-4 x y
$$

and

$$
L \equiv\left(a_{0} a_{2}-a_{1}^{2}\right) x-\left(a_{1} a_{3}-a_{-2}^{2}\right) y .
$$

Since the tangents at $U^{\prime}, V^{\prime}, W^{\prime}$ meet at $Q$, we see that $L$ is part of the polar conic of $Q$ with respect to the alove apolar locus. Hence the polar conic of $Q$ passes through $H$, shewing also that the polar line of $H$ passes through $Q . Q$ is therefore defined as the point of intersection of the polar line of $H$ with $M$. Now the polar line of $H$ with respect to (3) is

$$
\left(a_{0}-a_{3}\right)\left\{2 a_{2} x+2 a_{1} y+\left(a_{0}+a_{3}\right) z\right\}=6\left\{\left(a_{0} a_{2}-a_{1}^{2}\right) x-\left(a_{1} a_{3}-a_{2}^{3}\right) y\right\} .
$$

The symmetry of this result $(t)$ with regard to the invariants and covariants involved shews that it is also the polar line of $K$.

It is possible to identify geometrically the line (4) in the following way. There are four points on $\Gamma$ (viz., the Jacolian-tetrad) whose polar conics with respect to the triangles $A^{\prime} B^{\prime} C^{\prime}$ and $D^{\prime} E^{\prime} F^{\prime}$ intersect on $\Gamma$ itself. The conic locus $J$ passing through these four points and apolar to the conic envelope $\Gamma$ is

$$
J \equiv 6 a_{1} x^{2}-6 a_{2} y^{2}+\left(a_{3}-a_{0}\right) z^{2}-6 a_{1} y z+6 a_{2} z x+2\left(a_{3}-a_{0}\right) x y=0 .
$$

Also there are two points on $\Gamma$ whose polar lines with respect to the triangles $A^{\prime} B^{\prime} C^{\prime}, D^{\prime} E^{\prime} F^{\prime}$ are conjugate with respect to $\Gamma$. If the tangents 
at these two points meet at $O$, we have

$$
O \equiv\left\{a_{1}, a_{2},-\left(a_{0}+a_{8}\right)\right\} .
$$

By means of (5) and (6), it is easy to deduce that the polar line of $H$ (or $K$ ) with respect to the apolar locus of $\left(A^{\prime} B^{\prime} C^{\prime}, D^{\prime} E^{\prime} F^{\prime}\right)$ is the polar of the point $O$ with respect to the conic $J$.

The point on the line $M$ which corresponds to the apolar locus of $\left[A^{\prime} B^{\prime} C^{\prime}, D^{\prime} E^{\prime} F^{\prime}\right]$ according to the above method has therefore been identified geometrically.

We have therefore this final result:-

Let two apolar triads $A B C, D E F$ be projected through a suitable point $T$ into the apolar tricds $A^{\prime} B^{\prime} C^{\prime}, D^{\prime} E^{\prime} F^{\prime}$, so that the six points thus obtained lie on the same conic $\Gamma$. Let $L$ be the line joining the poles $H, K$ of $\Gamma$ with respect to the triangles $A^{\prime} B^{\prime} C^{\prime}, D^{\prime} E^{\prime} F^{\prime}$, and let the cubic intersect $L$ in $U^{\prime}, V^{\prime}, W^{\prime}$. Let $M$ be the line joining the poles of $L$ with respect to the triangles $A^{\prime} B^{\prime} C^{\prime}, D^{\prime} E^{\prime} F^{\prime}$. Let the $J$-conic and the O-point be defined as in (5) and (6) above with respect to the conic $\Gamma$ and the triads $A^{\prime} B^{\prime} C^{\prime}, D^{\prime} E^{\prime} F^{\prime}$ lying on $\Gamma$. Then the necessary and sufficient condition that the apolar triads $A B C, D E F$ be co-apolar is that the point of concuirency of the tangents to the cubic at $U^{\prime}, V^{\prime}, W^{\prime}$ be at the point of intersection of the polar of $O$ with respect to the conic $J$, and $M$ the line joining the poles of $L$ with respect to the triangles $A^{\prime} B^{\prime} C^{\prime}$ and $D^{\prime} E^{\prime} F^{\prime}$. 